\title{
Characterizing the conformational free-energy landscape of RNA us- ing single-molecule field-effect transistors
}

\author{
Sukjin S. Jang ${ }^{\ddagger}$, Sarah Dubnik ${ }^{\ddagger}$ Jason Hon ${ }^{\dagger}$, Colin Nuckolls*, and Ruben L. Gonzalez, Jr.* \\ Department of Chemistry, Columbia University, 3000 Broadway, New York, NY 10027, USA
}

KEYWORDS RNA, RNA stem-loop, RNA folding, RNA conformational dynamics, single-molecule biophysics, carbon nanotube, fieldeffect transistor

\begin{abstract}
We have developed and used high-time-resolution, single-molecule field-effect transistors (smFETs) to characterize the conformational free-energy landscape of RNA stem-loops. Stem-loops are some of the most common RNA structural motifs and serve as building blocks for the formation of more complex RNA structures. Given their prevalence and integral role in RNA folding, the kinetics of stem-loop (un)folding has been extensively characterized using both experimental and computational approaches. Interestingly, these studies have reported vastly disparate timescales of (un)folding, which has been recently interpreted as evidence that (un)folding of even simple stem-loops occurs on a highly rugged conformational energy landscape. Because smFETs do not rely on fluorophore reporters of conformation or on the application of mechanical (un)folding forces, they provide a unique and complementary approach that has allowed us to directly monitor tens of thousands of (un)folding events of individual stem-loops at a 200 $\mu$ s time resolution. Our results show that under our experimental conditions, stem-loops fold and unfold over a 1-200 ms timescale during which they transition between ensembles of unfolded and folded conformations, the latter of which is composed of at least two sub-populations. The 1-200 ms timescale of (un)folding we observe here indicates that smFETs report on complete (un)folding trajectories in which relatively extended unfolded conformations of the RNA spend long periods of time wandering the free-energy landscape before sampling one of several misfolded conformations or, alternatively, the natively folded conformation. Our findings demonstrate how the combination of single-molecule sensitivity and high time resolution makes smFETs unique and powerful tools for characterizing the conformational free-energy landscape of RNA and highlight the extremely rugged landscape on which even the simplest RNA structural elements fold.
\end{abstract}

\section{INTRODUCTION}

In this article, we report a method for tethering a single RNA molecule onto a nanoscopic electrical device that allows us to monitor (un)folding and/or structural rearrangements of the RNA across a broad range of timescales. RNA plays crucial roles in almost all essential biological functions. To perform these functions, RNAs must fold into complex three-dimensional structures and often undergo functionally important conformational changes across a wide range of timescales. ${ }^{1-3}$ One of the most fundamental and widely dispersed RNA structural elements are stem-loops, relatively small and fast-folding structures with highly variable thermodynamic stabilities ${ }^{4-6}$ (Figure 1a). Stem-loops commonly serve as folding nuclei for the formation of more complex RNA structural motifs. ${ }^{7}$ Additionally, they often participate in vital interactions with other RNAs or proteins and are frequently observed to undergo structural rearrangements that regulate such interactions..$^{1-2,8-10}$ Given such fundamental roles in RNA structure, dynamics, and function, the kinetics of stem-loop (un)folding have long been the subject of extensive characterization using both experimental and computational approaches. Notably, studies using different experimental techniques report disparate stem-loop (un)folding timescales, ranging from $10 \mu \mathrm{s}$ to $1 \mathrm{~s},{ }^{11-25}$ and disagree on whether intermediate states are observed during (un)folding. ${ }^{11,13-17,19,26}$ Recent computational studies suggest these inconsistencies originate from the fact that RNAs (un)fold on 'rugged' conformational free-energy landscapes containing a large number of energetic wells that are separated by many small and large barriers, and that different techniques simply sample distinct regions of these landscapes. ${ }^{17,27-29}$

Here, we develop, validate, and demonstrate the use of singlewalled carbon nanotube (SWCNT)-based, single-molecule field-effect transistor (smFET) devices for kinetic studies of RNA (un)folding and structural rearrangements. smFETs enable high-time-resolution, single-molecule studies of biomolecular dynamics that, uniquely, do not rely on fluorophore reporters of conformational changes nor on the application of mechanical force using an optical trapping instrument or atomic force microscope (AFM). ${ }^{30-39}$ Using this approach, we report the direct observation of conformational transitions in single RNA molecules across the microseconds-to-minutes timescales that are relevant for studies of RNA (un)folding and conformational dynamics. This includes transitions across the 1-200 ms timescales that would be exceedingly difficult, if not impossible, to simultaneously monitor at single-molecule resolution and in a fluorophore- and force-free manner. When the (un)folding of a single RNA molecule is monitored at these timescales and conditions, we find that the thousands of reversible (un)folding events that are observed for even simple stemloop structures exhibit significant kinetic heterogeneities. Moreover, our results strongly suggest that at least one source of these heterogeneities is the ability of the RNA to arrive at either the natively folded conformation or several misfolded conformations as it folds along a rugged conformational free- 
energy landscape. Collectively, the results of our studies demonstrate how the combination of high time resolution, single-molecule sensitivity, and fluorophore- and force-free operation of smFETs makes them a unique and powerful tool for characterizing the conformational free-energy landscapes that govern RNA (un)folding and structural rearrangements.

a

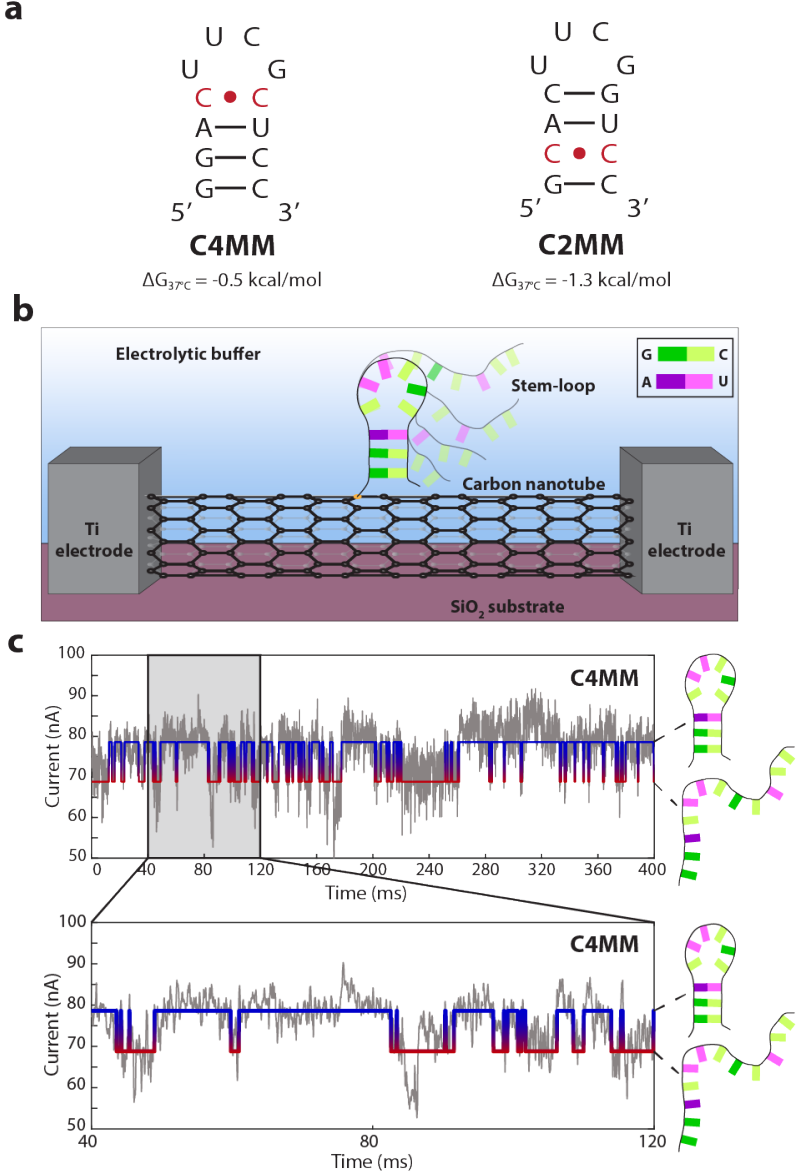

Figure 1. smFET experimental setup. (a) The two stem-loop constructs studied here each introduce a single mismatched bas-pair within the stem of the stem-loop by substituting a cytosine for a guanine. (b) A schematic cartoon of the smFET experimental platform is shown. Each smFET device consists of a SWCNT that serves as a conductive channel between a source- and drain electrode, with a stem-loop construct tethered to the surface of the SWCNT. Details of device fabrication and characterization are described in the Supporting Information and Figure S1. (c) A representative, 400-ms current versus time trajectory recorded for the C4MM construct, with an 80-ms subsection magnified below. These trajectories were collected at $200 \mu$ s time resolution. An idealized current state versus time trajectory (blue and red), obtained by applying a previously described two-state, drift-corrected, thresholding algorithm ${ }^{31-32,40}$ is overlaid on the current versus time trajectory. Details of the data analysis procedures are described in the Supporting Information.

\section{RESULTS AND DISCUSSION}

smFETs can monitor the (un)folding of a single RNA stemloop across a microseconds-to-minutes range of timescales. We designed two RNA stem-loop constructs as the targets of our study, each composed of a four base-pair stem and a wellcharacterized, thermodynamically stable UUCG tetraloop. ${ }^{41-42}$ The stem of each of these constructs contained a single nonWatson-Crick, $\mathrm{C} \cdot \mathrm{C}$ mismatched base-pair at either the fourth or second base-pair from the 5' and 3' ends of the stem-loop (referred to as the C4MM and C2MM constructs, respectively; see Figure 1a with mismatched base-pairs denoted in red). These mismatched base-pairs were engineered into the stems in order to destabilize the constructs such that they would be expected to readily undergo reversible (un)folding reactions within the temperature range accessible to our smFET devices (up to 50 ${ }^{\circ} \mathrm{C}$ ). The corresponding theoretical free-energy differences between the folded and unfolded states $(\Delta \mathrm{G})$ at $37^{\circ} \mathrm{C}\left(\Delta \mathrm{G}_{37^{\circ} \mathrm{C}}\right)$, predicted using the Tinoco and Turner nearest-neighbor energy rules, ${ }^{43-46}$ are listed beneath each construct. The 5' end of each construct was functionalized with a primary amine group (Table S1) that was used to tether the RNA to the surface of the SWCNTs, as described below and in the Supporting Information.

To tether the constructs to the SWCNT surface of the smFETs, the 5'-amine-functionalized RNAs were reacted with a carboxylic acid succinimidyl ester-derivatized pyrene (pyrene-NHS) that had been pre-tethered onto the SWCNT surface via noncovalent stacking of the pyrene moiety onto the SWCNT surface $\mathrm{e}^{35,38,47}$ (Figure 1b). The reaction conditions were optimized such that, on average, no more than one stem-loop was tethered to the SWCNT surface of an smFET (discussed in the following section). Tethering of an individual C4MM or C2MM stemloop to a single smFET resulted in single-molecule current versus time trajectories such as that shown in Figure 1c. Visual inspection of these trajectories revealed that both stem-loops transition rapidly between multiple current states. Observation of these transitions and states depended on a stem-loop having been tethered to the smFET (compare Figure S2 to Figure S3) and, once a stem-loop had been tethered, the observed transition frequencies were clearly temperature dependent (Figure S2b-c). A parsimonious analysis of these trajectories using a previously described two-state, drift-corrected, thresholding algorithm ${ }^{31-32}$, ${ }^{40}$ separated the observed states into a cluster of high current states (blue portion of the idealized path depicted in Figure 1c) and a cluster of low current states (red portion). Previous studies utilizing the smFET platform for biomolecular sensing have shown that the naturally occurring, electrostatically charged chemical groups of a single biomolecule tethered to the SWCNT surface of an smFET device locally gate the current transduction through the SWCNT. ${ }^{30-35,37-38,48}$ Consequently, as the tethered biomolecule (un)folds or undergoes a structural rearrangement, changes in the positions of the charged groups relative to the SWCNT surface result in changes to the local gating and corresponding conductivity of the smFET device. More specifically, these studies have demonstrated that relatively closer positioning of negatively charged moieties to the surface of the SWCNT results in relatively higher currents. ${ }^{31-32,35,37-38 \text {, }}$ ${ }^{48}$ Given the negatively charged phosphate groups of our stemloop constructs, we tentatively assigned the cluster of higher current states that we observed to an ensemble of folded conformations that would position a relatively larger number of phosphate groups closer to the SWCNT surface. Correspondingly, we assigned the cluster of lower current states to an ensemble of unfolded conformations. To confirm these assignments and characterize the (un)folding of the stem-loops, we first sought to validate that the observed trajectories originated from smFETs onto which a single stem-loop construct had been tethered to the SWCNT surface, and we subsequently characterized the temperature dependence of the observed transitions between the higher and lower current states.

Trajectories originate from single stem-loops that have been tethered to single smFETs. Several technical challenges have 
thus far prevented the use of smFET devices for studies of nucleic acid (un)folding and conformational dynamics. Perhaps the most problematic has been the difficulty of ensuring that only a single nucleic acid is tethered to the surface of a single smFET device. Previous efforts have focused on utilizing covalent tethering approaches and controlling this covalent chemistry to achieve single-molecule tethering. ${ }^{31-32,34}$ Here, we focus on utilizing a non-covalent tethering approach ${ }^{38,49}$ and developing a method to identify single nucleic acid molecules tethered to SWCNTs without perturbing the structural and/or electronic properties of the SWCNT. To characterize the number of nucleic acid molecules tethered to single smFETs and optimize our tethering protocol so as to ensure that only one molecule was tethered to each individual device, we developed a robust, AFM-based method for visualizing smFET-tethered nucleic acid constructs with single-molecule resolution. Evidence of tethering was facilitated by the formation of nucleic acid-protein complexes that functioned as proxies for nucleic acid-only constructs, which are themselves too small to be confidently visualized using contemporary AFM. We then characterized the number, locations, and specificity with which these complexes tethered to an smFET as a function of varying several parameters of our tethering protocol.

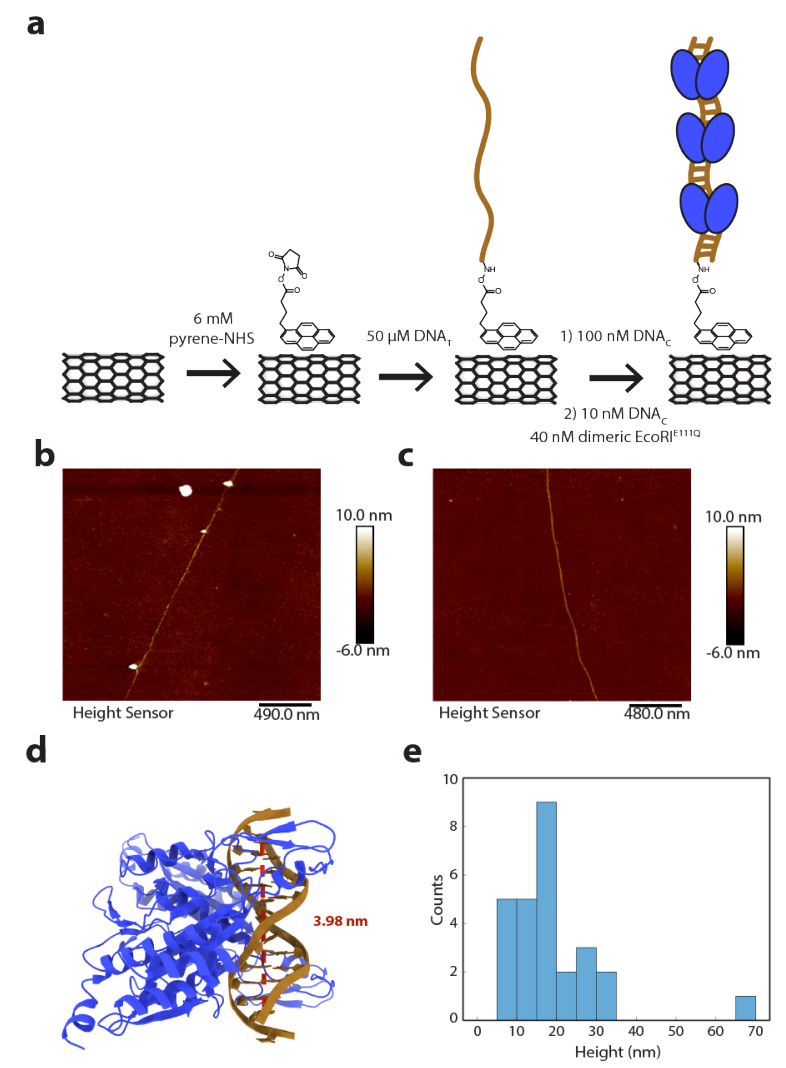

Figure 2. Quantifying and optimizing nucleic acid tethering conditions for smFET experiments. (a) Schematic cartoon of our tripleEcoRI ${ }^{\mathrm{E} 111 \mathrm{Q}}$-dimer-bound DNA complex tethering strategy. (b-c) smFET devices were imaged with AFM such that EcoRI ${ }^{\mathrm{E} 111 \mathrm{Q}}$-dimer-bound DNA complexes were visible as tall features on individual SWCNTs. (b) Representative AFM image of an smFET device containing 3 tethered EcoRI ${ }^{\mathrm{E} 111 \mathrm{Q}}$-dimer-bound DNA complexes generated following the strategy shown in (a). (c) Representative AFM image of an smFET device containing no tethered EcoRI ${ }^{\text {E111Q }}{ }^{-d i m e r-b o u n d ~ D N A ~ c o m p l e x e s ~ g e n e r a t e d ~ f o l l o w i n g ~ t h e ~}$ strategy shown in (a) with the exception that DNA $\mathrm{T}_{\mathrm{T}}$ was left out of the reaction as a negative control. (d) X-ray crystallographic struc-

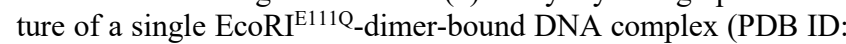
1ERI), used to determine the length of double-stranded DNA that is occluded by a single EcoRI ${ }^{\mathrm{E} 111 \mathrm{Q}}$ dimer. (e) Height distribution of EcoRI ${ }^{\mathrm{E} 11 \mathrm{Q}}$-dimer-bound DNA complexes observed in AFM images across three separate experiments.

We began by validating the specific tethering of pyrene onto SWCNTs using amine-modified gold nanoparticles. These experiments are described in the Supporting Information and Figure S4. Next, we used the pyrene-NHS incubation protocol to tether 5'-amine-modified, single-stranded, tethering DNA $\left(\mathrm{DNA}_{\mathrm{T}}\right)$ containing recognition sites for a previously described variant of the EcoRI homodimeric restriction endonuclease (Table S2), in which the glutamic acid at residue position $111 \mathrm{had}$ been mutated to a glutamine $\left(\mathrm{EcoRI}^{\mathrm{E} 111 \mathrm{Q}}\right) .{ }^{50} \mathrm{EcoRI}^{\mathrm{E} 111 \mathrm{Q}}$ is incapable of hydrolyzing its target DNA recognition sequence, instead binding to it with an extremely low equilibrium dissociation constant $\left(K_{\mathrm{d}}\right)$ of $2.5 \mathrm{fM} .^{50-51}$ Protein purification and characterization are detailed in the Supporting Information and Figure S5. After tethering of $\mathrm{DNA}_{\mathrm{T}}$ to the smFETs, single-stranded complementary DNA (DNA $)$ was introduced, followed by EcoRI $^{\mathrm{E} 111 \mathrm{Q}}$ in the presence of additional DNA $\mathrm{D}_{\mathrm{C}}$. Once hybridized, $\mathrm{DNA}_{\mathrm{T}}$ and $\mathrm{DNA}_{\mathrm{C}}$ formed a double-stranded DNA containing three tandem copies of the EcoRI ${ }^{\mathrm{E} 111 \mathrm{Q}}$ recognition site, each separated from the other by a 10 base-pair spacer so as to minimize the possibility that binding of one EcoRI ${ }^{\mathrm{E} 111 \mathrm{Q}}$ would sterically occlude binding of additional EcoRI ${ }^{\mathrm{E} 111 \mathrm{Q}_{\mathrm{S}}}$ to the remaining sites (Figure 2a). Binding of six copies (three dimers) of the $31-\mathrm{kDa}$ EcoRI $^{\mathrm{E} 111 \mathrm{Q}}$ thus allowed the triple-EcoRI ${ }^{\mathrm{E} 111 \mathrm{Q}}$-dimerbound, double-stranded DNA to be easily imaged and resolved using AFM (Figure 2b). As expected, control tethering experiments performed in the absence of $\mathrm{DNA}_{\mathrm{T}}$, but in the presence of DNA ${ }_{C}$ and EcoRI ${ }^{\mathrm{E} 111 \mathrm{Q}}$, did not produce the tethered, tripleEcoRI $^{\mathrm{E} 111 \mathrm{Q}}$-dimer-bound DNA complexes that were observed in the tethering experiments performed in the presence of $\mathrm{DNA}_{T}$ (compare Figure 2c with Figure 2b).

Given the relatively high concentrations of $\mathrm{DNA}_{\mathrm{C}}$ and EcoRI $^{\mathrm{E} 111 \mathrm{Q}}$ that were used in these experiments (10 nM and 40 $\mathrm{nM}$, respectively), the extremely low $K_{\mathrm{d}}$ for EcoRI ${ }^{\mathrm{E} 111 \mathrm{Q}}$ binding to its recognition sites, and the 10 base-pair separation between each of the three EcoRI ${ }^{\mathrm{E} 111 \mathrm{Q}}$ binding sites, we expected to maximize the number of $\mathrm{DNA}_{T} \mathrm{~S}$ that would be hybridized with DNA $_{C}$ and harbor three copies of dimeric EcoRI ${ }^{\mathrm{E} 111 \mathrm{Q}}$. As shown in the structure in Figure $2 d,{ }^{52}$ a single homodimer of EcoRI $^{\mathrm{E} 111 \mathrm{Q}}$ occludes a 3.98-nm, $\sim 12$ base-pair length of DNA containing a single recognition site. Considering the persistence length of DNA to be approximately $50 \mathrm{~nm},{ }^{53}$ the 12 base-pair length of DNA was extrapolated to a 48 base-pair, doublestranded B-form DNA helix containing three target sites:

$$
48 \text { base pairs } \times \frac{3.98 \mathrm{~nm}}{12 \text { base pairs }}=15.92 \mathrm{~nm}
$$

Consistent with this, the length distribution of the individual molecular constructs that were imaged using AFM exhibited a strong correspondence to the expected $\sim 16 \mathrm{~nm}$ length of a fully assembled, triple-EcoRI ${ }^{\mathrm{E} 111 \mathrm{Q}}$-dimer-bound DNA complex (Figure 2e). We therefore concluded that our AFM-based technique successfully enabled us to quantifiably visualize the number and locations of individual, triple-EcoRI ${ }^{\mathrm{E} 111 \mathrm{Q}}$-dimer-bound, DNA complexes, presumably reflecting the number and locations of individual $\mathrm{DNA}_{\mathrm{T}} \mathrm{S}$ on the surface of a single smFET device. 

available under aCC-BY-NC-ND 4.0 International license.

a

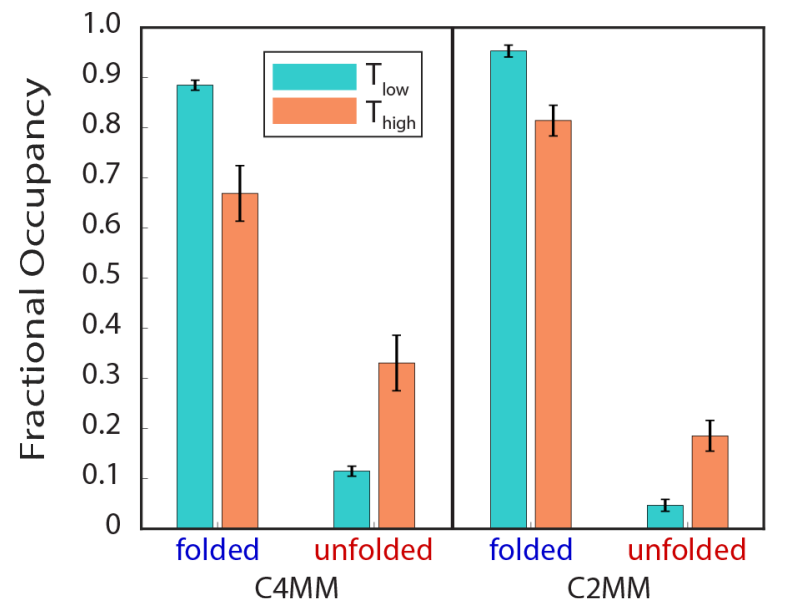

b

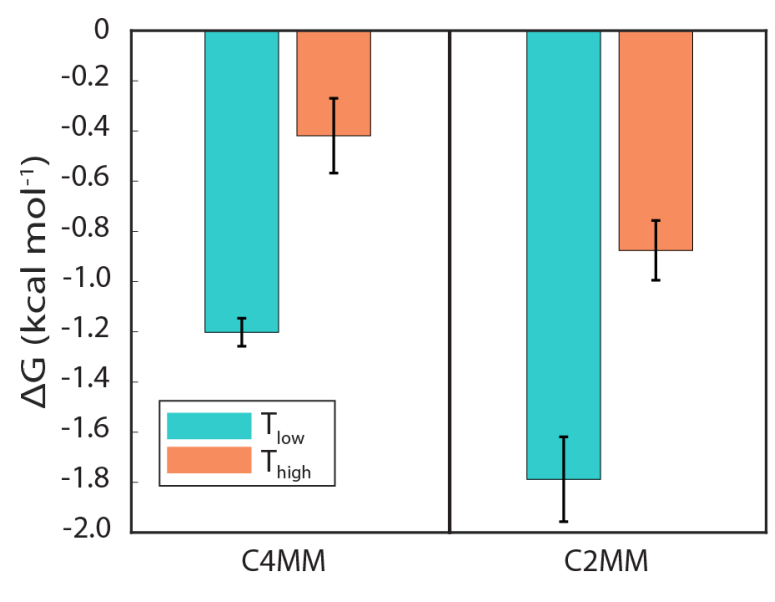

Figure 3. Thermodynamic analyses of $\mathrm{C} 4 \mathrm{MM}$ and $\mathrm{C} 2 \mathrm{MM}$ current versus time trajectories. (a) Plots of the fractional occupancies of the ensembles of folded and unfolded conformations at $\mathrm{T}_{\text {low }}\left(23-24{ }^{\circ} \mathrm{C}\right)$ and $\mathrm{T}_{\text {high }}\left(44-45^{\circ} \mathrm{C}\right)$ for $\mathrm{C} 4 \mathrm{MM}$ and $\mathrm{C} 2 \mathrm{MM}$. (b) Plots of the $\Delta \mathrm{Gs}$ of folding at $\mathrm{T}_{\text {low }}\left(23-24^{\circ} \mathrm{C}\right)$ and $\mathrm{T}_{\text {high }}\left(44-45^{\circ} \mathrm{C}\right)$ for $\mathrm{C} 4 \mathrm{MM}$ and $\mathrm{C} 2 \mathrm{MM}$. Error bars represent the standard deviations of the fractional occupancies and $\Delta$ Gs calculated from each of three separate 60-s windows of each current versus time trajectory.

Having validated our AFM-based approach for characterizing the number and locations of individual $\mathrm{DNA}_{\mathrm{T}} \mathrm{S}$ tethered to a single smFET, we used this method as a readout for varying several of the parameters of our tethering protocol (e.g., the concentration of pyrene-NHS; concentration of 5'-amine-functionalized DNA $\mathrm{T}_{\mathrm{T}}$; incubation times; washing steps and solvents; etc.) to identify the optimal values of these parameters that were most likely to result in tethering of a single DNA $_{T}$ per smFET. To this end, we found that the optimal conditions included incubating the smFETs in $6 \mathrm{mM}$ pyrene-NHS dissolved in dimethylformamide (DMF), followed by rinsing the smFETs twice with $25 \mu \mathrm{L}$ of DMF before introducing $50 \mu \mathrm{M}$ of $\mathrm{DNA}_{\mathrm{T}}$. These conditions resulted in tethering of approximately one $\mathrm{DNA}_{\mathrm{T}}$ per $5.79 \mu \mathrm{m}$ of SWCNT. Given that each smFET is comprised of a SWCNT with a length of approximately $2 \mu \mathrm{m}$, a Poisson distribution analysis of tethering under these conditions (see Supporting Information) results in $70.8,24.4$, and $4.2 \%$ probabilities of zero, one, and two tethered $\mathrm{DNA}_{\mathrm{T}} \mathrm{S}$, respectively, per device. In other words, given a device with a tethered $\mathrm{DNA}_{\mathrm{T}}$, there is an $83.9 \%$ probability of having a single tethered $\mathrm{DNA}_{\mathrm{T}}$. Furthermore, the observed distribution of the number of tethering per $2 \mu \mathrm{m}$ of SWCNT in Figure S6 agrees with the Poisson statistics described here.

Thermodynamic analyses of the trajectories validate our state assignments. To confirm that transitions between the clusters of high and low current states that we observed report on transitions between ensembles of folded and unfolded stemloop conformations, respectively, we used our smFET devices and optimized tethering strategy to characterize the thermodynamic stabilities, expressed in terms of $\Delta \mathrm{Gs}$, of the C4MM and $\mathrm{C} 2 \mathrm{MM}$ stem-loops. Because an increase in temperature is expected to destabilize the folded conformations and, as a consequence, increase the $\Delta \mathrm{G}$, an experimental demonstration that increasing the temperature increases the $\Delta \mathrm{G}$ would confirm that we have correctly assigned the clusters of high and low current states to ensembles of folded and unfolded conformations, respectively.

We began by recording a 10 min long trajectory for each of the $\mathrm{C} 4 \mathrm{MM}$ and $\mathrm{C} 2 \mathrm{MM}$ constructs at a relatively lower temperature of $23-24{ }^{\circ} \mathrm{C}$ ( $\mathrm{T}_{\text {low }}$ for the two constructs) as well as a relatively higher temperature of $44-45^{\circ} \mathrm{C}\left(\mathrm{T}_{\text {high }}\right)$ and analyzing each tra- jectory with our thresholding algorithm (see Supporting Information and Figures S2,3). The four trajectories had signal-tonoise ratios (SNRs) of 3-4 and, as expected, all four exhibited transitions between the clusters of high and low current states that were tentatively assigned to ensembles of folded and unfolded conformations, respectively, based on the sensing mechanism of the smFETs. To determine the putative $\triangle \mathrm{Gs}$ of C4MM and $\mathrm{C} 2 \mathrm{MM}$ at each temperature, we first quantified the total number of timepoints spent in the presumed ensembles of folded and unfolded conformations ( $t_{\mathrm{f}}$ and $t_{\mathrm{u}}$, respectively) over a representative, 60-s window of each trajectory and calculated the fractional occupancies of the folded and unfolded conformations $\left(f_{\mathrm{f}}=t_{\mathrm{f}} /\left(t_{\mathrm{f}}+t_{\mathrm{u}}\right)\right.$ and $f_{\mathrm{u}}=t_{\mathrm{u}} /\left(t_{\mathrm{f}}+t_{\mathrm{u}}\right)$, respectively) (Figure $3 a)$. These fractional occupancies were then used to calculate the putative equilibrium constants for the folding reactions ( $K$ $\left.=f_{\mathrm{f}} / f_{\mathrm{u}}\right)$ and $\Delta \mathrm{Gs}(\Delta \mathrm{G}=-R T \ln K$, where $R$ is the universal gas constant, $1.987 \mathrm{cal} / \mathrm{K} \cdot \mathrm{mol}$, and $T$ is the temperature at which the measurement was made).

As can be seen in Figure $3 \mathrm{~b}$, the $\Delta \mathrm{Gs}$ for $\mathrm{C} 4 \mathrm{MM}$ and $\mathrm{C} 2 \mathrm{MM}$ both increase, from $-1.20 \mathrm{kcal} / \mathrm{mol}$ and $-1.79 \mathrm{kcal} / \mathrm{mol}$, respectively, at $\mathrm{T}_{\text {low }}$, to $-0.42 \mathrm{kcal} / \mathrm{mol}$ and $-0.88 \mathrm{kcal} / \mathrm{mol}$, respectively, at $T_{\text {high }}$. The fact that the $\Delta \mathrm{Gs}$ for both constructs increase with increasing temperature confirms that we have correctly assigned the clusters of high and low current states observed in the trajectories to ensembles of folded and unfolded stem-loop conformations, respectively; mis-assignment of these states would have resulted in decreases to the $\Delta \mathrm{Gs}$ as a function of increasing temperature, which is not possible.

For C4MM and C2MM, the nearest-neighbor energy rules ${ }^{43-46}$ predict $\Delta \mathrm{G}_{37^{\circ} \mathrm{CS}}$ of $-0.5 \mathrm{kcal} / \mathrm{mol}$ and $-1.3 \mathrm{kcal} / \mathrm{mol}$ respectively, values that, for each construct, fall within the range of our experimentally measured $\Delta \mathrm{Gs}$ at $23-24^{\circ} \mathrm{C}$ and $44-45^{\circ} \mathrm{C}(-$ 1.20 and $-0.42 \mathrm{kcal} / \mathrm{mol}$, respectively, for $\mathrm{C} 4 \mathrm{MM}$ and -1.79 and $-0.88 \mathrm{kcal} / \mathrm{mol}$, respectively, for $\mathrm{C} 2 \mathrm{MM}$ ). The excellent agreement between our experimentally determined $\Delta \mathrm{Gs}$ and the $\Delta \mathrm{G}_{37^{\circ} \mathrm{CS}}$ predicted using the nearest-neighbor energy rules provide strong, further validation of our state assignments.

Kinetic analyses of the trajectories reveal the ruggedness of the stem-loop (un)folding free-energy landscape. A powerful advantage that smFETs bring to studies of RNA dynamics is that they enable interrogation of the conformational trajectories 
bioRxiv preprint doi: https://doi.org/10.1101/2022.01.13.475405; this version posted January 14, 2022. The copyright holder for this preprint (which was not certified by peer review) is the author/funder, who has granted bioRxiv a license to display the preprint in perpetuity. It is made available under aCC-BY-NC-ND 4.0 International license.

a
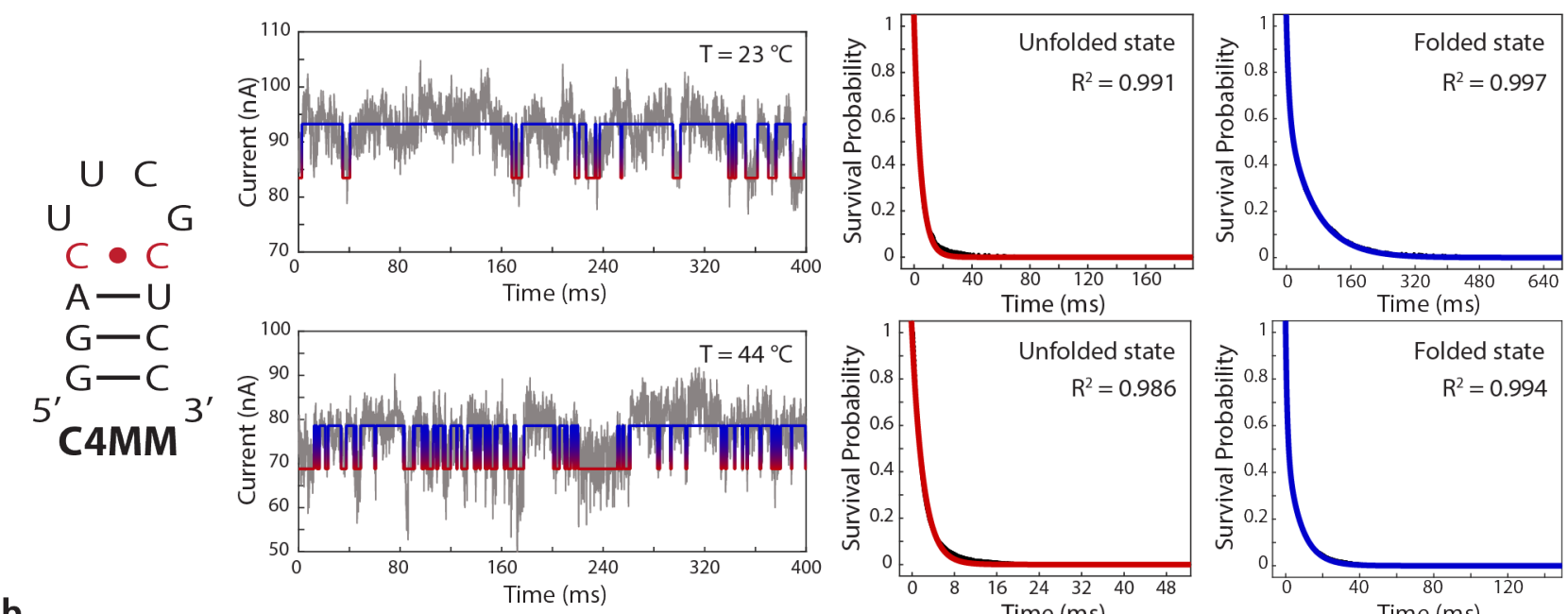

b
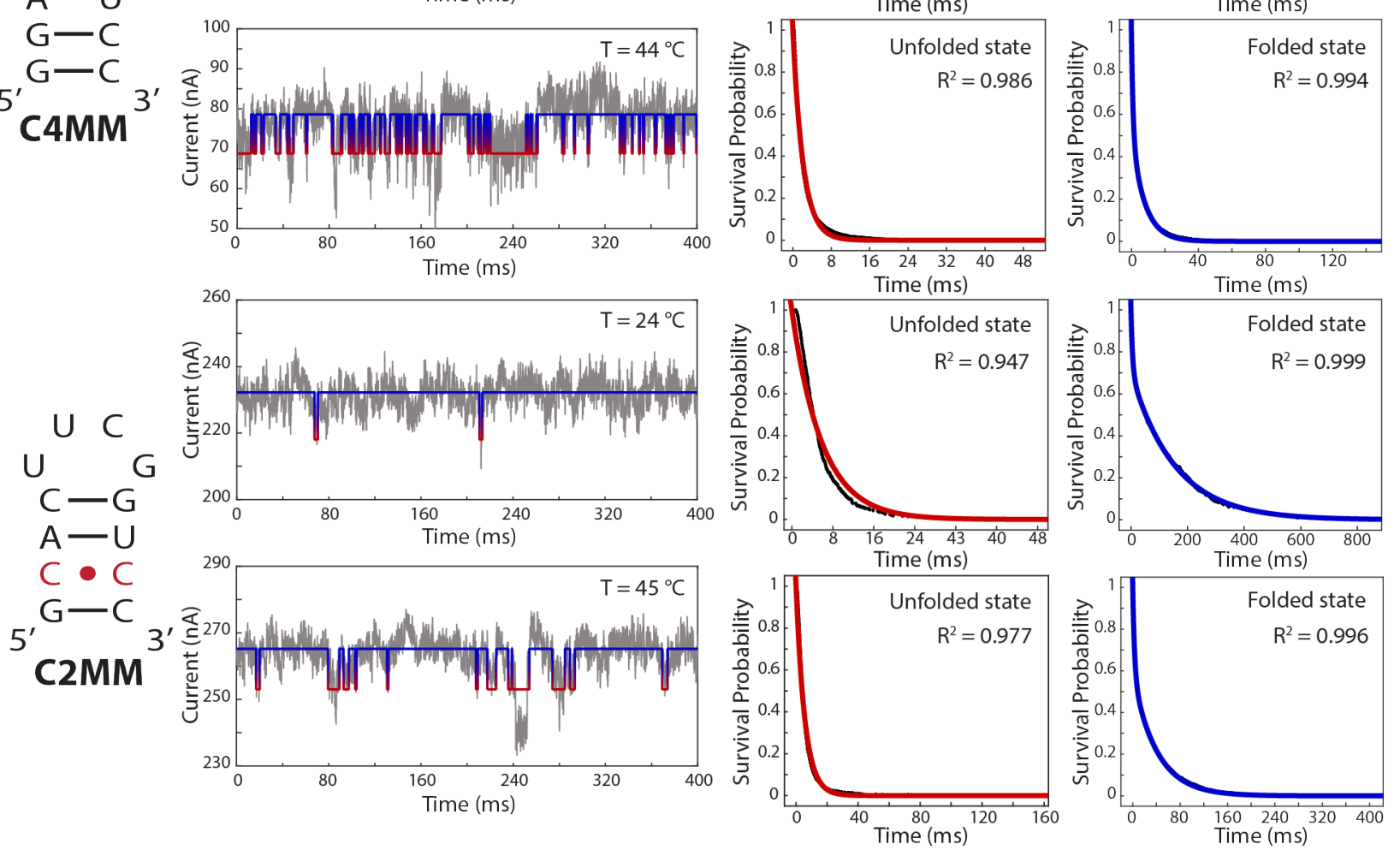

Figure 4. Kinetic analyses of C4MM and C2MM current versus time trajectories. Secondary structure diagrams of the stem-loop constructs with the mismatched base-pairs denoted in red (first column), representative current versus time trajectories (gray) with overlaid idealized current state versus time trajectories (blue and red) (second column), single-exponential fits (red curves) to the survival probability plots of the ensemble of unfolded conformations (black data points) at $\mathrm{T}_{\text {low }}$ (third column, top) and $\mathrm{T}_{\text {high }}$ (third column, bottom), and double-exponential fits (blue curves) to the survival probability plots of the ensemble of folded conformations (black data points) at $T_{\text {low }}$ (fourth column, top) and $\mathrm{T}_{\text {high }}$ (fourth column, bottom) for (a) C4MM and (b) C2MM.

of individual RNA molecules at an unprecedentedly high time resolution without the need for fluorophore reporters or the application of mechanical force. This property of smFETs potentially provides an avenue for detailed characterization of the ruggedness of the RNA conformational free-energy landscape. To probe this landscape for the (un)folding reactions of the $\mathrm{C} 4 \mathrm{MM}$ and $\mathrm{C} 2 \mathrm{MM}$ constructs, we performed kinetic analyses of our trajectories.

We began by analyzing the kinetics of transitions between the ensembles of folded and unfolded conformations as defined by our thresholding algorithm (see Supporting Information).Specifically, for C4MM and C2MM at each temperature, we determined the rates of folding and unfolding $\left(k_{\mathrm{f}}\right.$ and $k_{\mathrm{u}}$, respectively) by fitting exponential decay functions to plots of the survival probabilities of the ensembles of unfolded and folded conformations, respectively; the decay lifetimes for the ensemble of unfolded and folded conformations ( $\tau_{\mathrm{u}}$ and $\tau_{\mathrm{f}}$, respectively) were then used to calculate the rates $\left(k_{\mathrm{f}}=1 / \tau_{\mathrm{u}}\right.$ and $\left.k_{\mathrm{u}}=1 / \tau_{\mathrm{f}}\right)$. In all four cases (C4MM and C2MM at each temperature), a single-exponential decay was the simplest function that was needed to properly describe each plot of the survival probability of the ensemble of unfolded conformations, yielding folding rates $\left(k_{\mathrm{f}}\right)$ in the range of $\sim 150$ to $\sim 500 \mathrm{~s}^{-1}\left(\mathrm{R}^{2}=0.991-0.947\right.$; see Figure 4 and Table 1). The $k_{\mathrm{f}}$ for both C4MM and C2MM increased with increasing temperature (from $\sim 200$ to $\sim 500 \mathrm{~s}^{-1}$ for C4MM and from $\sim 150$ to $\sim 200 \mathrm{~s}^{-1}$ for C2MM), consistent with the expectation that the rate of biomolecular (un)folding processes should increase with increasing temperature.

In contrast, a double-exponential decay was the simplest function that could adequately describe the analogous plots of the survival probability in the ensemble of folded conformations $\left(\mathrm{R}^{2}=0.999-0.994\right.$ for double exponential fits vs $\mathrm{R}^{2}=0.928$ 0.832 for single-exponential fits; see Figure 4 and Figure S7, Supporting Information). Thus, at each temperature, C4MM and $\mathrm{C} 2 \mathrm{MM}$ can sample at least two different sub-populations of conformations that our thresholding algorithm collectively assign to the ensemble of folded conformations. In all cases, the longer-lived sub-population that unfolds with the relatively lower unfolding rate $\left(k_{\mathrm{u} \text {,slow }}\right)$ in the range of $\sim 10$ to $\sim 150 \mathrm{~s}^{-1} \mathrm{ex}$ hibits the higher fractional occupancy, in the range of $55-72 \%$ 
bioRxiv preprint doi: https://doi.org/10.1101/2022.01.13.475405; this version posted January 14, 2022. The copyright holder for this preprint (which was not certified by peer review) is the author/funder, who has granted bioRxiv a license to display the preprint in perpetuity. It is made available under aCC-BY-NC-ND 4.0 International license.

Table 1. Folding and unfolding rate constants of C4MM and C2MM at the measured temperatures. Rate constants are calculated from the survival probability plots of unfolded and folded state lifetimes. ${ }^{a}$

\begin{tabular}{c|cccccc}
\hline \multicolumn{2}{c}{ Temperature $\left({ }^{\circ} \mathbf{C}\right)$} & $\boldsymbol{k}_{\mathrm{f}}\left(\mathbf{s}^{-1}\right)$ & $\boldsymbol{k}_{\mathrm{u}, \text { fast }}\left(\mathbf{s}^{-1}\right)$ & $\begin{array}{c}\text { Fractional } \\
\text { Occupancy }(\%)\end{array}$ & $\begin{array}{c}\boldsymbol{k}_{\mathrm{u}, \mathrm{slow}} \\
\left(\mathbf{s}^{-1}\right)\end{array}$ & $\begin{array}{c}\text { Fractional } \\
\text { Occupancy }(\%)\end{array}$ \\
\hline \multirow{2}{*}{ C4MM } & 23 & $240(29)$ & $228(78)$ & $38(3)$ & $19(5)$ & $62(3)$ \\
& 44 & $486(38)$ & $983(271)$ & $45(5)$ & $146(58)$ & $55(5)$ \\
C2MM & 24 & $155(23)$ & $152(26)$ & $28(4)$ & $6(2)$ & $72(4)$ \\
& 45 & $160(20)$ & $315(26)$ & $41(1)$ & $22(3)$ & $59(1)$
\end{tabular}

${ }^{a}$ Rate constants and (standard deviations) were calculated using the average of three separate $60 \mathrm{~s}$ windows from each trajectory.

(Figure 4, Table 1). Contrasting with this, the shorter-lived subpopulation that unfolds with the relatively faster unfolding rate $\left(k_{\mathrm{u}, \text { fast }}\right)$ in the range of $\sim 150$ to $\sim 1000 \mathrm{~s}^{-1}$ exhibits the lower fractional occupancy, in the range of $28-45 \%$ (Figure 4, Table 1). Again as expected, $k_{\mathrm{u}, \text { slow }}$ and $k_{\mathrm{u} \text {, fast }}$ for both C4MM and C2MM increased with increasing temperature (from $\sim 20$ to $\sim 150 \mathrm{~s}^{-1}$ and from $\sim 10$ to $\sim 20 \mathrm{~s}^{-1}$ for $k_{\mathrm{u}, \text { slow }}$ for C4MM and C2MM, respectively, and from $\sim 200$ to $\sim 1000 \mathrm{~s}^{-1}$ and from $\sim 150$ to $\sim 300 \mathrm{~s}^{-1}$ for $k_{\mathrm{u} \text {,fast }}$ for C4MM and C2MM, respectively). Moreover, for both C4MM and C2MM, the fractional occupancy of the longer-lived, slower unfolding sub-population decreases with increasing temperature (from 62 to $55 \%$ for $\mathrm{C} 4 \mathrm{MM}$ and from 72 to $59 \%$ for $\mathrm{C} 2 \mathrm{MM}$ ), suggesting that one of the mechanisms through which $k_{\mathrm{u}}$ increases as a function of increasing temperature is via destabilization of the most stable sub-population of folded conformations. A possible interpretation of the identities of these sub-populations of folded conformations can be drawn from previous theoretical studies of RNA stem-loop (un)folding kinetics. ${ }^{17,54-56}$ Specifically, these studies propose that the ratelimiting step in the stem-loop folding process is the diffusive search across the conformational free-energy landscape for an RNA conformation that is aligned so as to nucleate formation of interactions that are found in the natively folded stem-loop conformation (i.e., native interactions), while also sampling numerous RNA conformations that form non-native interactions. Formation of such non-native interactions can frequently result in misfolded structures that may or may not be able to proceed to the natively folded stem-loop conformation. In the context of our smFET studies, we hypothesize that our shorter-lived, faster unfolding sub-population of folded conformations represents a collection of misfolded conformations in which non-native interactions persist for a relatively short, but detectable timescale before becoming disrupted so as to allow unfolding of the misfolded conformation. Correspondingly, our longer-lived, slower unfolding sub-population of folded conformations represents the natively folded stem-loop conformation. Future smFET studies focusing on comprehensively elucidating the mechanism of stem-loop (un)folding could use variations of the sequence and length of the stem and loop to further confirm the identity of the two sub-populations of folded conformations and characterize how these sub-populations contribute to the mechanism of stem-loop (un)folding.

In order to further integrate our findings into current models of RNA stem-loop (un)folding, we next turned to a set of ensemble T-jump studies of RNA stem-loop constructs similar to C4MM and C2MM. ${ }^{11-14}$ Notably, our smFET measurements demonstrate that the C4MM and C2MM stem-loops undergo (un)folding transitions on 1-200 ms timescales, whereas ensemble Tjump experiments show that similar stem-loops undergo relax- ation processes on faster, 1-100 $\mu \mathrm{s}$, timescales. This discrepancy of up to three orders-of-magnitude in timescales most likely arises from a fundamental difference in how smFET and ensemble T-jump experiments probe the conformational freeenergy landscape. Specifically, on average, the divergence between the starting and ending conformations on the landscape probed in a single smFET measurement is greater than that of a single ensemble T-jump measurement. For example, unfolding events observed during an smFET experiment might start from the natively folded conformation and end in a relatively extended unfolded conformation. Contrasting with this, ensemble T-jump experiments probe the landscape by applying a small, instantaneous change in temperature to a conformationally equilibrated population and subsequently recording how the population relaxes to a new equilibrium position. Consequently, an ensemble T-jump experiment that starts from a population predominantly composed of the natively folded conformation will necessarily end at a population that is only slightly more unfolded. Because they involve starting and ending conformations that are more divergent, smFET experiments probe (un)folding processes that occur on slower timescales than those that are probed by ensemble T-jump experiments. This interpretation is supported by recent computational studies by Chakraborty et al. ${ }^{27}$ showing that (un)folding timescales strongly depend on the identities of the starting and ending conformations that are probed by different experimental techniques.

Although any one ensemble T-jump experiment probes only a small region of the conformational free-energy landscape, one way of extending the reach of these studies is to perform a series of experiments in which each experiment starts from a different conformationally equilibrated population (i.e., a different starting temperature). When such studies were performed on RNA stem-loops similar to C4MM and C2MM, the relaxation processes recorded at multiple starting temperatures could be globally fit to a model in which the natively folded stem-loop conformation proceeded to a fully unfolded conformation via two intermediate states, with transitions between all four states occurring on 1-100 $\mu$ s timescales. ${ }^{11,13-14}$ Although our trajectories might sample similar, short-lived intermediate states, the 200 $\mu$ s time resolution of our smFET experiments would preclude us from directly observing them. Currently, we are developing a complementary smFET platform with improved circuit board design and device architecture that is expected to increase our time resolution by two or more orders-of-magnitude and should allow us to directly detect and characterize such short-lived intermediates.

Despite having analyzed our data using a simplified, two-state thresholding algorithm, visual inspection of the trajectories reveals that individual excursions into the low- and high-current 

available under aCC-BY-NC-ND 4.0 International license.

states that the algorithm collectively assigns as the unfolded and folded conformations, respectively, differ in the absolute value of the observed current. Given the low-frequency ' $1 / f$ ' noise of our smFETs ${ }^{31-32}$ and the relatively low SNR of our trajectories, we cannot rule out the possibility that at least some of the variations in the current values within the low- and high-current states are due to these sources of noise. Nonetheless, it is possible that at least some of the excursions to low- and high-current states with different current values represent sampling of distinct unfolded and folded conformations, respectively. Ongoing and future improvements of the smFET technology and/or data analysis algorithms (see below) should ultimately allow us to discern additional, authentic conformational states from device noise.

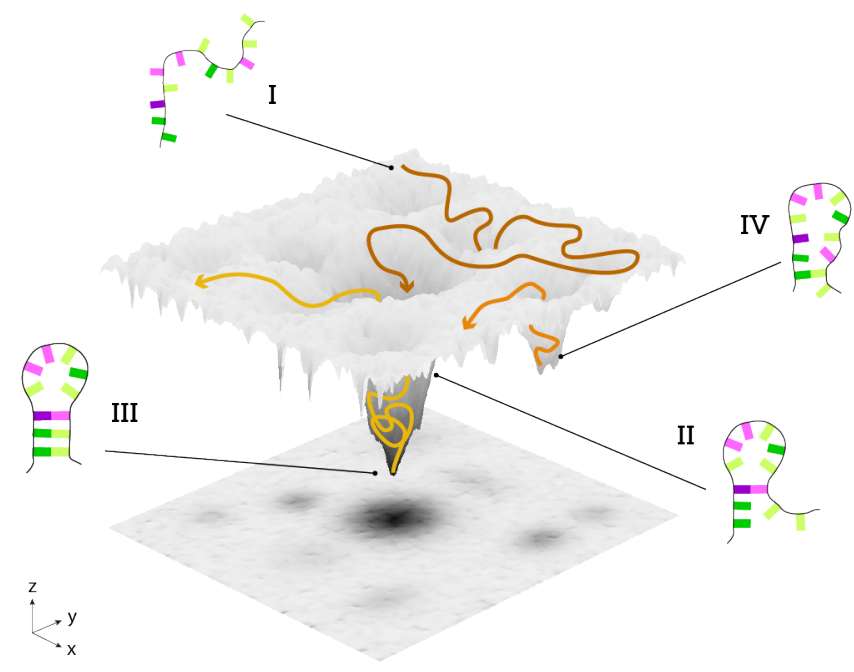

Figure 5. Schematic cartoon of a three-dimensional projection of the multi-dimensional conformational free-energy landscape onto representative RNA stem-loop (un)folding reaction coordinates. Arrows on the landscape depict three representative pathways of (un)folding. The X- and Y dimensions represent reaction coordinates representing different stem-loop conformations and the $\mathrm{Z}$ dimension represents the conformational free energy. See Supporting Information for details.

\section{CONCLUSIONS}

We have developed a new, robust, smFET-based approach for single-molecule studies of RNA (un)folding and structural rearrangements that provides data on timescales of 1-200 ms. Furthermore, we have used this approach to investigate the kinetics of RNA stem-loop (un)folding. Our results provide direct evidence for transitions between ensembles of unfolded and folded conformations and reveal heterogeneities in the unfolding kinetics, suggesting that the folded population consists of natively folded and misfolded conformations. Figure 5 integrates our results and the results of the previous ensemble experimental and computational studies described above as a schematic cartoon of a three-dimensional projection of the multi-dimensional conformational free-energy landscape onto representative RNA stem-loop (un)folding reaction coordinates. As expected from previous studies, the landscape is rugged, featuring many energetic wells corresponding to sets of non-native interactions that act as 'kinetic traps' before the RNA samples a nucleating native interaction that allows the RNA to rapidly fold into the stem-loop conformation. In the figure, the dark brown pathway represents a folding event from an smFET experiment in which the RNA initiates from a relatively extended unfolded conformation (Conformation I) and samples a collapsed state containing a nucleating native interaction (Conformation II) that allows it to form the natively folded stem-loop conformation (Conformation III) on a 1-10 ms timescale. We note here that the rate of transitions between Conformations II and III, which can be inferred from relaxation processes observed in ensemble T-jump experiments, are too fast for us to resolve given the timescale of our smFET measurements. The orange pathway represents an unfolding event in which an RNA that has formed non-native interactions and fallen into a kinetic trap (Conformation IV) escapes from the trap on a relatively fast timescale of $1-10 \mathrm{~ms}$ to continue its diffusive search across the landscape. The gold pathway, on the other hand, represents an unfolding event in which an RNA that has formed the natively folded stem-loop conformation unfolds on a relatively slow timescale of 10-200 ms.

When taken together, the results of our experiments and those performed in the previous experimental and computational investigations highlight how the (un)folding kinetics of stemloops are strongly dependent on the starting and ending conformations and emphasize the complementary nature of results obtained from these different techniques. The sensing mechanism and single-molecule nature of smFET experiments, for example, uniquely enable fluorophore- and force-free direct observation of individual, complete folding trajectories that start from relatively extended unfolded conformations and end in the natively folded conformation, trajectories that exhibit relatively slow kinetics. They also uniquely allow detection and parsing of molecular sub-populations that exhibit different folding behaviors. The high time resolution of ensemble T-jump experiments, on the other hand, uniquely enable inference of shortlived intermediate conformations and the rate constants connecting such conformations to the unfolded and folded conformations. Ongoing and future development of the smFET technology described here should enable even more powerful studies of RNA (un)folding and conformational dynamics. An important direction, for example, is the development of smFETs with improved circuit board design and device architecture that will push the time resolution to $1-10 \mu$ s and enable us to directly observe and characterize short-lived intermediate conformations currently embedded within the observed ensemble of unfolded and folded conformations. Such developments also promise improvements in the $1 / f$ noise and SNRs of smFETs, which will enable the development of more sophisticated, probabilistic data analysis methods that will enable more comprehensive analysis of the resulting current versus time trajectories. Similarly, ongoing and future extensions of the proof-of-principle RNA stem-loop (un)folding experiments reported here should allow a mechanistically richer understanding of stemloop (un)folding. Specifically, varying the lengths and sequences of the stem and loop components of our stem-loop should allow us to further characterize the heterogeneity we have identified in the ensemble of folded conformations, elucidating how these variables contribute to the efficiency with which stem-loops fold from their unfolded conformations into the natively folded conformation instead of any of the several misfolded conformations.

Most importantly, the smFET-based experimental framework for single-molecule studies of RNA (un)folding and conformational dynamics we describe here is entirely generalizable. It can be easily extended to investigate the mechanisms of (un)folding of RNA molecules adopting other stem-loop structures, (un)folding of RNA molecules that fold into structures more complex than simple stem-loops, structural rearrangements of RNA molecules that function as conformational 

available under aCC-BY-NC-ND 4.0 International license.

switches, and even the interactions of RNA-binding proteins with their RNA targets. In each of these cases, fluorophore- and force-free, single-molecule studies that simultaneously monitor timescales across microseconds to minutes are expected to provide mechanistic details that have been difficult or impossible to obtain using other single-molecule or ensemble approaches. Such developments would bring the field of single-molecule biophysics closer to one of its grand challenges: correspondence between the timescales of single-molecule experiments and molecular dynamics (MD) simulations of those experiments. Such correspondence should allow MD-based, atomic-resolution interpretations of the conformational dynamics captured by single-molecule experiments as well as the experimental testing of MD-derived hypotheses of how conformationally dynamic processes contribute to RNA function.

\section{ASSOCIATED CONTENT}

\section{Supporting Information}

Materials and methods used to fabricate and characterize devices, optimize tethering approach, purify and characterize EcoRI ${ }^{\mathrm{E} 111 \mathrm{Q}}$, obtain stem-loop current traces, analyze data, and generate the conformational free-energy landscape figure are described. Figures illustrate device characterization (Figure S1), I-V and current versus time traces (Figures S2,3), specificity of pyrene adsorption (Figure S4), EcoRI ${ }^{\mathrm{E} 11 \mathrm{Q}}$ digestion assay gel (Figure S5), distribution of number of $\mathrm{DNA}_{\mathrm{T}}$ tethers per device (Figure S6), and additional survival probability exponential curves (Figure S7). Tables describe DNA and RNA constructs used in smFET experiments (Table S1) and AFM-based imaging (Table S2). This material is available free of charge via the Internet at http://pubs.acs.org.

\section{AUTHOR INFORMATION}

\section{Corresponding Authors}

* Colin Nuckolls, Department of Chemistry, Columbia University, 3000 Broadway, New York, NY 10027, USA, Tel: (212) 854-6289, ORCHID: orcid.org/0000-0002-0384-5493, Email: cn37@columbia.edu; Ruben L. Gonzalez, Jr., Department of Chemistry, Columbia University, 3000 Broadway, New York, NY 10027, USA, Tel: (212) 854-1096, ORCHID: orcid.org/00000002-1344-5581, Email: rlg2118@,columbia.edu

\section{Present Addresses}

$\uparrow$ Department of Systems Biology, Harvard Medical School, Boston, MA, USA

\section{Author Contributions}

All authors have given approval to the final version of the manuscript. / $\$$ These authors contributed equally.

\section{Funding}

This research was supported by the National Science Foundation (CHE 2004016) and the National Institutes of Health (GM107417).

\section{Notes}

The authors declare no competing financial interest.

\section{ACKNOWLEDGMENT}

C.N. thanks Sheldon and Dorothea Buckler for their generous support. This research was supported by the National Science Foundation (CHE 2004016) and the National Institutes of Health (GM107417). We thank Scott Trocchia for providing the electronic board for the smFETs; Erik Young for providing guidance on building our temperature control system; Kevin Renehan and Eric Pollman for helping with wire-bonding; Riley C. Gentry, Nicholas
Ide, and Erik Hartwick for providing guidance during protein purification; Scott Trocchia, Colin Kinz-Thompson, and Korak K. Ray for assistance with data analysis and generation of energy landscape figure. This work was carried out in part in the Clean Room, Electron Microscopy, and Shared Materials Characterization labs of Columbia Nano Initiative (CNI) Shared Lab Facilities at Columbia University, and the Precision Biomolecular Characterization Facility (PBCF). Essential instrumentation in the PBCF was made possible by funding from the U.S. National Institutes of Health (S10OD025102). We thank Jia Ma for management of the PBCF.

\section{REFERENCES}

1. Mustoe, A. M.; Brooks, C. L.; Al-Hashimi, H. M., Hierarchy of RNA functional dynamics. Annu Rev Biochem 2014, 83, 44166.

2. Ganser, L. R.; Kelly, M. L.; Herschlag, D.; Al-Hashimi, H. M., The roles of structural dynamics in the cellular functions of RNAs. Nat Rev Mol Cell Biol 2019, 20 (8), 474-489.

3. Brion, P.; Westhof, E., Hierarchy and dynamics of RNA folding. Annu Rev Biophys Biomol Struct 1997, 26, 113-37.

4. Gutell, R. R., Collection of small subunit (16S- and 16S-like) ribosomal RNA structures. Nucleic Acids Res 1993, 21 (13), 3051-4.

5. Wolters, J., The nature of preferred hairpin structures in $16 \mathrm{~S}$ like rRNA variable regions. Nucleic Acids Res 1992, 20 (8), 1843-50.

6. Antao, V. P.; Lai, S. Y.; Tinoco, I., Jr., A thermodynamic study of unusually stable RNA and DNA hairpins. Nucleic Acids Res 1991, 19 (21), 5901-5.

7. Tinoco, I., Jr.; Bustamante, C., How RNA folds. J Mol Biol 1999, 293 (2), 271-81.

8. Bevilacqua, P. C.; Blose, J. M., Structures, kinetics, thermodynamics, and biological functions of RNA hairpins. Annu Rev Phys Chem 2008, 59, 79-103.

9. Butcher, S. E.; Pyle, A. M., The molecular interactions that stabilize RNA tertiary structure: RNA motifs, patterns, and networks. Acc Chem Res 2011, 44 (12), 1302-11.

10. Thapar, R.; Denmon, A. P.; Nikonowicz, E. P., Recognition modes of RNA tetraloops and tetraloop-like motifs by RNAbinding proteins. Wiley Interdiscip Rev RNA 2014, 5 (1), 49-67.

11. Ma, H.; Proctor, D. J.; Kierzek, E.; Kierzek, R.; Bevilacqua, P. C.; Gruebele, M., Exploring the energy landscape of a small RNA hairpin. J Am Chem Soc 2006, 128 (5), 1523-30.

12. Proctor, D. J.; Ma, H.; Kierzek, E.; Kierzek, R.; Gruebele, M.; Bevilacqua, P. C., Folding thermodynamics and kinetics of YNMG RNA hairpins: specific incorporation of 8bromoguanosine leads to stabilization by enhancement of the folding rate. Biochemistry 2004, 43 (44), 14004-14.

13. Sarkar, K.; Meister, K.; Sethi, A.; Gruebele, M., Fast folding of an RNA tetraloop on a rugged energy landscape detected by a stacking-sensitive probe. Biophys $J$ 2009, 97 (5), 1418-27.

14. Sarkar, K.; Nguyen, D. A.; Gruebele, M., Loop and stem dynamics during RNA hairpin folding and unfolding. Rna 2010, 16 (12), 2427-34.

15. Stancik, A. L.; Brauns, E. B., Rearrangement of partially ordered stacked conformations contributes to the rugged energy landscape of a small RNA hairpin. Biochemistry 2008, 47 (41), 10834-40.

16. Narayanan, R.; Zhu, L.; Velmurugu, Y.; Roca, J.; Kuznetsov, S. V.; Prehna, G.; Lapidus, L. J.; Ansari, A., Exploring the energy landscape of nucleic acid hairpins using laser temperature-jump and microfluidic mixing. J Am Chem Soc 2012, 134 (46), 18952-63.

17. Kuznetsov, S. V.; Ansari, A., A kinetic zipper model with intrachain interactions applied to nucleic acid hairpin folding kinetics. Biophys J 2012, 102 (1), 101-11. 
bioRxiv preprint doi: https://doi.org/10.1101/2022.01.13.475405; this version posted January 14,2022 . The copyright holder for this preprint

(which was not certified by peer review) is the author/funder, who has granted bioRxiv a license to display the preprint in perpetuity. It is made available under aCC-BY-NC-ND 4.0 International license.

18. Kuznetsov, S. V.; Ren, C. C.; Woodson, S. A.; Ansari, A., Loop dependence of the stability and dynamics of nucleic acid hairpins. Nucleic Acids Res 2008, 36 (4), 1098-112.

19. Abdollah-Nia, F.; Gelfand, M. P.; Van Orden, A., Three-State DNA Hairpin Conformational Dynamics Revealed by HigherOrder Fluorescence Correlation Spectroscopy. J Phys Chem B 2019, 123 (7), 1491-1504.

20. Bonnet, G.; Krichevsky, O.; Libchaber, A., Kinetics of conformational fluctuations in DNA hairpin-loops. Proc Natl Acad Sci U S A 1998, 95 (15), 8602-6.

21. Grunwell, J. R.; Glass, J. L.; Lacoste, T. D.; Deniz, A. A.; Chemla, D. S.; Schultz, P. G., Monitoring the conformational fluctuations of DNA hairpins using single-pair fluorescence resonance energy transfer. J Am Chem Soc 2001, 123 (18), 4295-303.

22. Tsukanov, R.; Tomov, T. E.; Berger, Y.; Liber, M.; Nir, E., Conformational dynamics of DNA hairpins at millisecond resolution obtained from analysis of single-molecule FRET histograms. J Phys Chem B 2013, 117 (50), 16105-9.

23. Woodside, M. T.; Anthony, P. C.; Behnke-Parks, W. M.; Larizadeh, K.; Herschlag, D.; Block, S. M., Direct measurement of the full, sequence-dependent folding landscape of a nucleic acid. Science 2006, 314 (5801), 1001-4.

24. Woodside, M. T.; Behnke-Parks, W. M.; Larizadeh, K.; Travers, K.; Herschlag, D.; Block, S. M., Nanomechanical measurements of the sequence-dependent folding landscapes of single nucleic acid hairpins. Proc Natl Acad Sci U S A 2006, 103 (16), 6190-5.

25. Liphardt, J.; Onoa, B.; Smith, S. B.; Tinoco, I., Jr.; Bustamante, C., Reversible unfolding of single RNA molecules by mechanical force. Science 2001, 292 (5517), 733-7.

26. Nayak, R. K.; Peersen, O. B.; Hall, K. B.; Van Orden, A., Millisecond time-scale folding and unfolding of DNA hairpins using rapid-mixing stopped-flow kinetics. J Am Chem Soc 2012, 134 (5), 2453-6.

27. Chakraborty, D.; Collepardo-Guevara, R.; Wales, D. J., Energy landscapes, folding mechanisms, and kinetics of RNA tetraloop hairpins. J Am Chem Soc 2014, 136 (52), 18052-61.

28. Hyeon, C.; Thirumalai, D., Mechanical unfolding of RNA hairpins. Proc Natl Acad Sci U S A 2005, 102 (19), 6789-94.

29. Hyeon, C.; Thirumalai, D., Multiple probes are required to explore and control the rugged energy landscape of RNA hairpins. J Am Chem Soc 2008, 130 (5), 1538-9.

30. Sorgenfrei, S.; Chiu, C. Y.; Johnston, M.; Nuckolls, C.; Shepard, K. L., Debye screening in single-molecule carbon nanotube field-effect sensors. Nano Lett 2011, 11 (9), 3739-43.

31. Lee, Y.; Trocchia, S. M.; Warren, S. B.; Young, E. F.; Vernick, S.; Shepard, K. L., Electrically Controllable Single-Point Covalent Functionalization of Spin-Cast Carbon-Nanotube Field-Effect Transistor Arrays. ACS Nano 2018, 12 (10), 99229930.

32. Vernick, S.; Trocchia, S. M.; Warren, S. B.; Young, E. F.; Bouilly, D.; Gonzalez, R. L.; Nuckolls, C.; Shepard, K. L., Electrostatic melting in a single-molecule field-effect transistor with applications in genomic identification. Nat Commun 2017, 8,15450 .

33. Sorgenfrei, S.; Chiu, C. Y.; Gonzalez, R. L., Jr.; Yu, Y.J.; Kim, P.; Nuckolls, C.; Shepard, K. L., Label-free single-molecule detection of DNA-hybridization kinetics with a carbon nanotube field-effect transistor. Nat Nanotechnol 2011, 6 (2), 126-32.

34. Bouilly, D.; Hon, J.; Daly, N. S.; Trocchia, S.; Vernick, S.; Yu, J.; Warren, S.; Wu, Y.; Gonzalez, R. L.; Shepard, K. L.; Nuckolls, C., Single-Molecule Reaction Chemistry in Patterned Nanowells. Nano Letters 2016, 16 (7), 4679-4685.

35. Choi, Y.; Olsen, T. J.; Sims, P. C.; Moody, I. S.; Corso, B. L.; Dang, M. N.; Weiss, G. A.; Collins, P. G., Dissecting single- molecule signal transduction in carbon nanotube circuits with protein engineering. Nano Lett 2013, 13 (2), 625-31.

36. Olsen, T. J.; Choi, Y.; Sims, P. C.; Gul, O. T.; Corso, B. L.; Dong, C.; Brown, W. A.; Collins, P. G.; Weiss, G. A., Electronic measurements of single-molecule processing by DNA polymerase I (Klenow fragment). J Am Chem Soc 2013, 135 (21), 7855-60.

37. Akhterov, M. V.; Choi, Y.; Olsen, T. J.; Sims, P. C.; Iftikhar, M.; Gul, O. T.; Corso, B. L.; Weiss, G. A.; Collins, P. G., Observing lysozyme's closing and opening motions by highresolution single-molecule enzymology. ACS Chem Biol 2015, 10 (6), 1495-501.

38. Choi, Y.; Moody, I. S.; Sims, P. C.; Hunt, S. R.; Corso, B. L.; Perez, I.; Weiss, G. A.; Collins, P. G., Single-molecule lysozyme dynamics monitored by an electronic circuit. Science 2012, 335 (6066), 319-24.

39. Sims, P. C.; Moody, I. S.; Choi, Y.; Dong, C.; Iftikhar, M.; Corso, B. L.; Gul, O. T.; Collins, P. G.; Weiss, G. A., Electronic measurements of single-molecule catalysis by cAMPdependent protein kinase A. J Am Chem Soc 2013, 135 (21), 7861-8.

40. Plesa, C.; Dekker, C., Data analysis methods for solid-state nanopores. Nanotechnology 2015, 26 (8), 084003.

41. Antao, V. P.; Tinoco, I., Thermodynamic parameters for loop formation in RNA and DNA hairpin tetraloops. Nucleic acids research 1992, 20 (4), 819-824.

42. Antao, V. P.; Lai, S. Y.; Tinoco, I., A thermodynamic study of unusually stable RNA and DNA hairpins. Nucleic Acids Research 1991, 19 (21), 5901-5905.

43. Mathews, D. H.; Sabina, J.; Zuker, M.; Turner, D. H., Expanded sequence dependence of thermodynamic parameters improves prediction of RNA secondary structure. J Mol Biol 1999, 288 (5), 911-40.

44. Xia, T.; SantaLucia, J., Jr.; Burkard, M. E.; Kierzek, R.; Schroeder, S. J.; Jiao, X.; Cox, C.; Turner, D. H., Thermodynamic parameters for an expanded nearest-neighbor model for formation of RNA duplexes with Watson-Crick base pairs. Biochemistry 1998, 37 (42), 14719-35.

45. Zuker, M., Mfold web server for nucleic acid folding and hybridization prediction. Nucleic Acids Res 2003, 31 (13), 3406- 15 .

46. Tinoco, I., Jr.; Borer, P. N.; Dengler, B.; Levin, M. D.; Uhlenbeck, O. C.; Crothers, D. M.; Bralla, J., Improved estimation of secondary structure in ribonucleic acids. Nat New Biol 1973, 246 (150), 40-1.

47. Chen, R. J.; Zhang, Y.; Wang, D.; Dai, H., Noncovalent sidewall functionalization of single-walled carbon nanotubes for protein immobilization. J Am Chem Soc 2001, 123 (16), 3838-9.

48. Heller, I.; Janssens, A. M.; Männik, J.; Minot, E. D.; Lemay, S. G.; Dekker, C., Identifying the mechanism of biosensing with carbon nanotube transistors. Nano Lett 2008, 8 (2), 591-5.

49. Chen, R. J.; Bangsaruntip, S.; Drouvalakis, K. A.; Kam, N. W.; Shim, M.; Li, Y.; Kim, W.; Utz, P. J.; Dai, H., Noncovalent functionalization of carbon nanotubes for highly specific electronic biosensors. Proc Natl Acad Sci U S A 2003, 100 (9), 4984-9.

50. Wright, D. J.; King, K.; Modrich, P., The negative charge of Glu-111 is required to activate the cleavage center of EcoRI endonuclease. J Biol Chem 1989, 264 (20), 11816-21.

51. King, K.; Benkovic, S. J.; Modrich, P., Glu-111 is required for activation of the DNA cleavage center of EcoRI endonuclease. J Biol Chem 1989, 264 (20), 11807-15.

52. Kim, Y. C.; Grable, J. C.; Love, R.; Greene, P. J.; Rosenberg, J. M., Refinement of Eco RI endonuclease crystal structure: a revised protein chain tracing. Science 1990, 249 (4974), 13079. 
bioRxiv preprint doi: https://doi.org/10.1101/2022.01.13.475405; this version posted January 14,2022 . The copyright holder for this preprint (which was not certified by peer review) is the author/funder, who has granted bioRxiv a license to display the preprint in perpetuity. It is made available under aCC-BY-NC-ND 4.0 International license.

53. Hagerman, P. J., Flexibility of DNA. Annu Rev Biophys Biophys Chem 1988, 17, 265-86.

54. Zhang, W.; Chen, S. J., RNA hairpin-folding kinetics. Proc Natl Acad Sci U S A 2002, 99 (4), 1931-6.

55. Sorin, E. J.; Rhee, Y. M.; Pande, V. S., Does water play a structural role in the folding of small nucleic acids? Biophys $J$ 2005, 88 (4), 2516-24.
56. Sorin, E. J.; Rhee, Y. M.; Nakatani, B. J.; Pande, V. S., Insights into nucleic acid conformational dynamics from massively parallel stochastic simulations. Biophys $J$ 2003, 85 (2), 790803. 